This item was submitted to Loughborough's Research Repository by the author.

Items in Figshare are protected by copyright, with all rights reserved, unless otherwise indicated.

\title{
Argument shift method and sectional operators: applications to differential geometry
}

PLEASE CITE THE PUBLISHED VERSION

http://www.fdis2015.wfa.uz.zgora.pl/?q=node/7

\section{PUBLISHER}

FDIS

\section{VERSION}

VoR (Version of Record)

\section{PUBLISHER STATEMENT}

This work is made available according to the conditions of the Creative Commons Attribution-NonCommercialNoDerivatives 4.0 International (CC BY-NC-ND 4.0) licence. Full details of this licence are available at: https://creativecommons.org/licenses/by-nc-nd/4.0/

\section{LICENCE}

CC BY-NC-ND 4.0

\section{REPOSITORY RECORD}

Bolsinov, Alexey V.. 2019. "Argument Shift Method and Sectional Operators: Applications to Differential Geometry”. figshare. https://hdl.handle.net/2134/19946. 


\title{
Argument shift method and sectional operators: applications to differential geometry
}

\author{
Alexey Bolsinov \\ Loughborough University, UK \\ and \\ Moscow State University
}

FDIS 2015

13-17 July, Bedlewo, Poland 


\section{What is it about?}

Review on joint papers with V.Matveev, V.Kiosak, S.Rosemann, D.Tsonev and A.Konyaev 


\section{Pre-history}

Let $\mathfrak{g}$ be a semisimple Lie algebra, $R: \mathfrak{g}^{*} \simeq \mathfrak{g} \rightarrow \mathfrak{g}$ a symmetric linear operator. Euler equations on $\mathfrak{g}^{*}$

$$
\frac{d x}{d t}=[x, R(x)]
$$

are Hamiltonian with $H=\frac{1}{2}\langle R(x), x\rangle$.

For which $R$, are the equations (1) integrable?

\section{Definition}

$R: \operatorname{so}(n) \rightarrow \operatorname{so}(n)$ is called a sectional operator (with parameters $A$ and $B$ ), if

$$
[R(X), A]=[X, B] \quad \text { for all } X \in \operatorname{so}(g)
$$

where $A$ and $B$ are some fixed symmetric matrices.

Theorem (Manakov, Mischenko, Fomenko)

Let $R$ satisfy (2). Then

- (1) can be rewritten as $\frac{d}{d t}(X+\lambda A)=[X+\lambda A, R(X)+\lambda B]$;

- $\operatorname{Tr}(X+\lambda A)^{k}$ are commuting first integrals of (1);

- if $A$ is regular, then (1) are completely integrable. 


\section{Properties of sectional operators}

1. $A$ and $B$ commute, moreover, $B$ belongs to the centre of centraliser of $A$. In particular, $B=p(A)$, where $p(\cdot)$ is some polynomial.

2. $R_{0}=\left.\frac{d}{d t}\right|_{t=0} p(A+t X)$ satisfies (2). If $A$ is regular, then $R$ is unique, otherwise $R=R_{0}+D$ where $D: \operatorname{so}(g) \rightarrow g_{A}=\{Y \in \operatorname{so}(g), A Y=Y A\}$ is arbitrary.

3. if $B=0=p_{\min }(A)$, then $R_{0}=\left.\frac{d}{d t}\right|_{t=0} p_{\min }(A+t X)$ still defines a non-trivial sectional operator whose image is contained in $\mathfrak{g}_{A}$. Moreover, if for each eigenvalues of $A$ there are at most 2 Jordan blocks, then the image $R_{0}$ coincides with $\mathfrak{g}_{A}$.

4. $R_{0}$ satisfies the Bianchi identity.

5. Let $R$ satisfy two identities $[R(X), A]=[X, B]$ and $\left[R(X), A^{\prime}\right]=\left[X, B^{\prime}\right]$, where $A^{\prime} \neq a A+b \cdot$ id. Then $R(X)=k \cdot X \bmod \mathfrak{g}_{A}$. In particular, if $A$ is regular, then $R=k \cdot$ id.

6. Let $\lambda_{1}, \ldots, \lambda_{k}$ be the eigenvalues of $A$. Then $\frac{p\left(\lambda_{i}\right)-p\left(\lambda_{j}\right)}{\lambda_{i}-\lambda_{j}}$ are eigenvalues of $R$. Moreover, if $A$ has a nontrivial Jordan $\lambda_{i}$-block, then $p^{\prime}\left(\lambda_{i}\right)$ is an eigenvalue of $R$. 


\section{Riemann curvature tensor (quick reminder and "new" point of view)}

Let $\nabla$ be the Levi-Civita connection of a Riemannian metric $g$.

Definition

The Riemann curvature tensor $R=\left(R_{i j}^{l} k\right)$ is defined by (formula from a text-book):

$$
R(X, Y) Z=\nabla_{X} \nabla_{Y} Z-\nabla_{Y} \nabla_{X} Z-\nabla_{[X, Y]} Z .
$$

In other words, $R$ can be understood as a map

$$
R:(X, Y) \mapsto R(X, Y)=\nabla_{X} \nabla_{Y}-\nabla_{Y} \nabla_{X}-\nabla_{[X, Y]} \in \operatorname{End}(T M) .
$$

Algebraic symmetries:

- $R(X, Y)=-R(X, Y)$, i.e., $R: \wedge^{2} V \rightarrow \operatorname{gl}(V), V=T_{X} M$;

- $g(R(X, Y) Z, W)=-g(R(X, Y) W, Z)$, i.e. $R(X, Y) \in \operatorname{so}(g)$;

- $R(X, Y) Z+R(Y, Z) X+R(Z, X) Y=0$ (Bianchi identity);

- $g(R(X, Y) Z, W)=-g(R(Z, W) X, Y)$.

Conclusion: $\quad R: \operatorname{so}(g) \rightarrow \operatorname{so}(g)$ which is symmetric and satisfying Bianchi. 


\section{Projectively equivalent metrics}

\section{Definition}

$g$ and $\bar{g}$ are projectively equivalent if they have the same (unparametrised) geodesics. Notation: $g \underset{\text { proj }}{\simeq} \bar{g}$.

Main equation: Let $A=\left(\frac{\operatorname{det} \bar{g}}{\operatorname{det} g}\right)^{\frac{1}{n+1}} \bar{g}^{-1} g$. Then $g \underset{\text { proj }}{\simeq} \bar{g}$ if and only if

$$
\nabla_{u} A=\frac{1}{2}\left(u \otimes d \operatorname{tr} A+(u \otimes d \operatorname{tr} A)^{*}\right) .
$$

Theorem (B., Matveev)

Let $g \underset{\text { proj }}{\simeq} \bar{g}$. Then the Riemann curvature tensor of $g$ is a sectional operator:

$$
[R(X), A]=[B, X] \quad \text { for all } X \in s o(g), \text { where } B=\frac{1}{2} \nabla(\operatorname{grad} \operatorname{tr} A) .
$$

Proof.

Consider the compatibility condition for the main equation.

Theorem (B., Matveev, Kiosak)

Let $g, \bar{g}$ and $\hat{g}$ be projectively equivalent. Assume that these metrics are linearly independent and $g$ and $\hat{g}$ are strictly non-proportional, then $g, \bar{g}$ and $\hat{g}$ are metrics of constant sectional curvature.

Proof.

Apply Property 5. 


\section{New class of holonomy groups in pseudo-Riemannian geometry}

\section{Definition}

Let $M$ be a smooth manifold endowed with an affine symmetric connection $\nabla$. The holonomy group of $\nabla$ is a subgroup $\operatorname{Hol}(\nabla) \subset \mathrm{GL}\left(T_{\times} M\right)$ that consists of the linear operators $A: T_{x} M \rightarrow T_{x} M$ being 'parallel transport transformations' along closed loops $\gamma(t)$ with $\gamma(0)=\gamma(1)=x$.

Problem. Given a subgroup $H \subset G L(n, \mathbb{R})$, can it be realised as the holonomy group for an appropriate symmetric connection on $M^{n}$ ?

Riemannian case and irreducible case: the problem is completely solved (Marcel Berger, D. V. Alekseevskii, R. Bryant, D. Joyce, L. Schwahhöfer, S. Merkulov).

Pseudo-Riemannian case: many fundamental results but still open (L. Bérard Bergery, A. Ikemakhen, C. Boubel, D. V. Alekseevskii, T. Leistner, A. Galaev).

Theorem (B., Tsonev)

For every $g$-symmetric operator $A: V \rightarrow V$, its centraliser in $\mathrm{SO}(g)$ (the identity connected component of)

$$
G_{A}=\{Y \in \mathrm{SO}(g) \mid Y A=A Y\}
$$

is a holonomy group for a certain (pseudo)-Riemannian metric. 


\section{Classical approach}

\section{Definition}

A map $R: \Lambda^{2} V \rightarrow \operatorname{gl}(V)$ is called a formal curvature tensor if it satisfies the Bianchi identity

$$
R(u \wedge v) w+R(v \wedge w) u+R(w \wedge u) v=0 \quad \text { for all } u, v, w \in V .
$$

\section{Definition}

Let $\mathfrak{h} \subset \operatorname{gl}(V)$ be a Lie subalgebra. Consider the set of all formal curvature tensors $R: \Lambda^{2} V \rightarrow \operatorname{gl}(V)$ such that $\operatorname{Im} R \subset \mathfrak{h}$ :

$\mathcal{R}(\mathfrak{h})=\left\{R: \Lambda^{2} V \rightarrow \mathfrak{h} \mid R(u \wedge v) w+R(v \wedge w) u+R(w \wedge u) v=0, u, v, w \in V\right\}$.

We say that $\mathfrak{h}$ is a Berger algebra if it is generated as a vector space by the images of the formal curvature tensors $R \in \mathcal{R}(\mathfrak{h})$, i.e.,

$$
\mathfrak{h}=\operatorname{span}\{R(u \wedge v) \mid R \in \mathcal{R}(\mathfrak{h}), u, v \in V\} .
$$

Berger test:

Let $\nabla$ be a symmetric affine connection on TM. Then the Lie algebra hol $(\nabla)$ of its holonomy group $\mathrm{Hol}(\nabla)$ is Berger. 


\section{Classical approach (with small amendments)}

Definition

A map $R: \mathrm{so}(g) \rightarrow \mathrm{so}(g)$ is called a formal curvature tensor if it satisfies the Bianchi identity

$$
R(u \wedge v) w+R(v \wedge w) u+R(w \wedge u) v=0 \quad \text { for all } u, v, w \in V,
$$

where $u \wedge v=u \otimes g(v)-v \otimes g(u) \in \operatorname{so}(g)$.

Definition

Let $\mathfrak{h} \subset \mathrm{so}(g)$ be a Lie subalgebra. Consider the set of all formal curvature tensors $R: \operatorname{so}(g) \rightarrow \operatorname{so}(g)$ such that $\operatorname{Im} R \subset \mathfrak{h}$ :

$\mathcal{R}(\mathfrak{h})=\left\{R: \Lambda^{2} V \rightarrow \mathfrak{h} \mid R(u \wedge v) w+R(v \wedge w) u+R(w \wedge u) v=0, u, v, w \in V\right\}$.

We say that $\mathfrak{h}$ is a Berger algebra if it is generated as a vector space by the images of the formal curvature tensors $R \in \mathcal{R}(\mathfrak{h})$, i.e.,

$$
\mathfrak{h}=\operatorname{span}\{R(u \wedge v) \mid R \in \mathcal{R}(\mathfrak{h}), u, v \in V\} .
$$

Berger test:

Let $\nabla$ be a symmetric affine connection on TM. Then the Lie algebra hol $(\nabla)$ of its holonomy group $\mathrm{Hol}(\nabla)$ is Berger. 


\section{Step one: Berger test for $\mathfrak{g}_{A}$ and Magic Formula 1}

We have

$$
\mathfrak{g}_{A}=\{X \in \operatorname{so}(g) \mid X A=A X\}
$$

and we need to construct formal curvature tensors $R: \operatorname{so}(g) \rightarrow \operatorname{so}(g)$ whose images generate $\mathfrak{g}_{A}$.

Ideally, we want one single formal curvature tensor $R$ such that $\operatorname{Im} R=\mathfrak{g}_{A}$.

Question: How to find $R$ ?

Answer: Apply Properties 3 and 4, i.e. define a linear mapping $R: \operatorname{so}(g) \rightarrow \operatorname{so}(g)$ by:

$$
R(X)=\left.\frac{d}{d t}\right|_{t=0} p_{\min }(A+t X),
$$

where $p_{\min }(\lambda)$ is the minimal polynomial of $A$.

Conclusion: $\mathfrak{g}_{A}$ is Berger algebra. 


\section{Step two: Realisation}

We need to find an example of $g$ such that $\mathfrak{h o l}(\nabla)=\mathfrak{g}_{A}$.

More specifically:

For a given operator $A: T_{x_{0}} M \rightarrow T_{x_{0}} M$, we need to find

a (pseudo)-Riemannian metric $g$ on $M$ and

a $(1,1)$-tensor field $A(x)$ (with the initial condition $A\left(x_{0}\right)=A$ ) such that

1. $\nabla A(x)=0$;

2. $R\left(x_{0}\right)$ coincides with the formal curvature tensor $R_{\text {formal }}$ just defined.

The idea is natural:

- set $A(x)=$ const

- try to find the desired metric $g(x)$ in the form:

$$
\text { constant }+ \text { quadratic }
$$

i.e.,

$$
g_{i j}(x)=g_{i j}^{0}+\sum \mathcal{B}_{i j, p q} x^{p} x^{q}
$$

where $\mathcal{B}$ satisfies obvious symmetry relations, namely, $\mathcal{B}_{i j, p q}=\mathcal{B}_{j i, p q}$ and $\mathcal{B}_{i j, p q}=\mathcal{B}_{i j, q p}$. 


\section{Magic Formula 2}

Thus, we need to find $\mathcal{B}_{i j, p q}$ with the required properties. Such a tensor can be rewritten in the form $\mathcal{B}=\sum \mathcal{C}_{\alpha} \otimes \mathcal{D}_{\alpha}$, where $\mathcal{C}_{\alpha}$ and $\mathcal{D}_{\alpha}$ are some symmetric forms. It is more convenient to work with "operators" rather than "forms":

$$
\mathcal{B}=\sum \mathcal{C}_{\alpha} \otimes \mathcal{D}_{\alpha} \quad \longrightarrow \quad B=\sum C_{\alpha} \otimes D_{\alpha},
$$

where $C_{\alpha}$ and $D_{\alpha}$ are the $g_{0}$-symmetric operators corresponding to $\mathcal{C}_{\alpha}$ and $\mathcal{D}_{\alpha}$. Then we can treat $B$ as a linear map

$$
B: \operatorname{gl}(V) \rightarrow \operatorname{gl}(V) \text { defined by } B(X)=\sum C_{\alpha} X D_{\alpha},
$$

Question: How to find $B$ ?

Answer: Amasingly simple $B=\frac{1}{2} R(\otimes)$, i.e.

$$
R(X)=\left.\frac{d}{d t}\right|_{t=0} p_{\min }(A+t X) \quad \mapsto \quad B=\left.\frac{1}{2} \cdot \frac{d}{d t}\right|_{t=0} p_{\min }(L+t \cdot \otimes),
$$

More precisely, if $p_{\min }(\lambda)=\sum_{m=0}^{n} a_{m} \lambda^{m}$ is the minimal polynomial of $A$, then

$$
B=\frac{1}{2} \cdot \sum_{m=0}^{n} a_{m} \sum_{j=0}^{m-1} A^{m-1-j} \otimes A^{j} .
$$

Conclusion: This $B$ solves the realisation problem. 


\section{C-projective equivalence and Yano-Obata conjecture}

Definition

A curve $\gamma(t)$ on a Kähler manifold $(M, g, J)$ is called $J$-planar, if

$$
\nabla_{\gamma} \dot{\gamma}=\alpha \dot{\gamma}+\beta J \dot{\gamma}
$$

where $\alpha, \beta \in \mathbb{R}$, and $J$ is the complex structure on $M$.

\section{Definition}

Two Kähler metrics $g$ and $\bar{g}$ on a complex manifold $(M, J)$ are called c-projectively equivalent, if they have the same $J$-planar curves.

\section{Definition}

A vector field $\xi$ on a Kähler manifold is called c-projective, if the flow of $\xi$ preserves $J$-planar curves. A c-projective vector field is called essential if its flow changes the Levi-Civita connection.

Theorem (B., Matveev, Rosemann)

Let $(M, g, J)$ be a closed connected Kähler manifold of arbitrary signature which admits an essential c-projective vector field. Then the manifold is isometric to $\mathbb{C} P^{n}$ with the Fubini-Study metric. 


\section{Some ingredients of the proof}

Statement 1 . Let $g$ and $\bar{g}$ be projectively equivalent Kähler metrics. Then the Riemann curvature tensor of $g$ satisfies

$$
[R(X), A]=[X, B] \quad \text { for all } X \in \mathrm{u}(g),
$$

where $A=\left(\frac{\operatorname{det} \bar{g}}{\operatorname{det} g}\right)^{\frac{1}{2(n+1)}} \bar{g}^{-1} g$ and $B=\nabla(\operatorname{grad} \operatorname{tr} A)$ (hermitian operators). In other words, $R$ can be considered as a sectional operator for the unitary algebra.

Statement 2. If $A$ admits a non-trivial Jordan block, then one of the eigenvalues of $R$ can be explicitly computed from Property 6 .

Statement 3. Let $x(t)$ be a trajectory of $\xi$. This eigenvalue $\lambda \in \operatorname{Spectrum}(R)$, as a function of $t$, i.e. $\lambda(x(t))$ is not bounded. Therefore, $M$ cannot be compact. 


\section{Thanks for your attention}

